\title{
Estimation of fractional cycle bias for GPS/ BDS-2/Galileo based on international GNSS monitoring and assessment system observations using the uncombined PPP model
}

\author{
Jin Wang, Qin Zhang ${ }^{*}$ and Guanwen Huang
}

\begin{abstract}
The Fractional Cycle Bias (FCB) product is crucial for the Ambiguity Resolution (AR) in Precise Point Positioning (PPP). Different from the traditional method using the ionospheric-free ambiguity which is formed by the Wide Lane (WL) and Narrow Lane (NL) combinations, the uncombined PPP model is flexible and effective to generate the FCB products. This study presents the FCB estimation method based on the multi-Global Navigation Satellite System (GNSS) precise satellite orbit and clock corrections from the international GNSS Monitoring and Assessment System (iGMAS) observations using the uncombined PPP model. The dual-frequency raw ambiguities are combined by the integer coefficients $(4,-3)$ and $(1,-1)$ to directly estimate the FCBs. The details of FCB estimation are described with the Global Positioning System (GPS), BeiDou-2 Navigation Satellite System (BDS-2) and Galileo Navigation Satellite System (Galileo). For the estimated FCBs, the Root Mean Squares (RMSs) of the posterior residuals are smaller than 0.1 cycles, which indicates a high consistency for the float ambiguities. The stability of the WL FCBs series is better than 0.02 cycles for the three GNSS systems, while the STandard Deviation (STD) of the NL FCBs for BDS-2 is larger than 0.139 cycles. The combined FCBs have better stability than the raw series. With the multi-GNSS FCB products, the PPP AR for GPS/BDS-2/Galileo is demonstrated using the raw observations. For hourly static positioning results, the performance of the PPP AR with the three-system observations is improved by $42.6 \%$, but only $13.1 \%$ for kinematic positioning results. The results indicate that precise and reliable positioning can be achieved with the PPP AR of GPS/BDS-2/Galileo, supported by multi-GNSS satellite orbit, clock, and FCB products based on iGMAS.
\end{abstract}

Keywords: PPP AR, Fractional cycle bias, Satellite orbit and clock products, iGMAS

\section{Introduction}

Precise Point Positioning (PPP) Ambiguity Resolution (AR) is important to obtain the positioning accuracy at centimeter-level in a short time at a station (Bisnath and Gao 2008; Wang et al. 2019). Due to the Fractional Cycle Biases (FCBs) in phase measurements which are assimilated into the undifferenced ambiguities in PPP, the integer properties of the estimated ambiguities are lost

*Correspondence: zhangqinle@263.net.cn

College of Geology Engineering and Geomatics, Chang'an University, Xi'an 710054, China
(Gabor and Nerem 1999). Currently, it is assumed that FCBs contain the biases from satellite and receiver hardware delays. Unlike the relative positioning model, where FCBs are eliminated in double-differenced Global Navigation Satellite System (GNSS) observations (Ge et al. 2008; Geng et al. 2012), the PPP processing needs the FCB products to correct the float ambiguities for recovering their integer properties.

The method of ambiguity resolution based on the satellite-satellite single differences was first proposed in PPP (Gabor and Nerem 1999), many methods have then been developed with different products to obtain the 
ambiguity-fixed positioning solutions (Ge et al. 2008; Collins et al. 2008; Laurichesse et al. 2009). Based on an empirical assumption that the Uncalibrated Phase Delays (UPD) are relatively stable in time, the SingleDifference (SD) FCBs of the Wide Lane (WL) and Narrow Lane (NL) float ambiguities between satellites are estimated from a reference network (Ge et al. 2008). The WL FCBs are determined by averaging the fractional parts of all WL ambiguities from the same satellites. The Melbourne-Wübbena (MW) measurements are used to derive the wide-lane ambiguities (Melbourne 1985; Wübbena 1985). Generally, the WL FCBs are stable over a few days, or even several months (Gabor and Nerem 1999). After determining the WL FCBs, the integer part of the $\mathrm{WL}$ ambiguities is used to estimate the float narrow-lane ambiguities. Similarly, the NL FCBs are also determined by averaging the fractional parts of the NL ambiguities. Due to the short wavelength of the narrow-lane ambiguity, for instance, about $10 \mathrm{~cm}$ for Global Positioning System (GPS), the NL FCBs are not as stable as the WL, which are proposed to estimate 15 min mean values.

Instead of estimating the SD FCB from the same satellite pairs, Li and Zhang (2012) proposed that the ZeroDifference (ZD) FCBs can be estimated together in the adjustment system using the least-square method with all FCB measurements (Li and Zhang 2012). This approach is adopted for the WL and NL FCBs estimation, which significantly improved the accuracy of estimated FCBs. For the NL FCBs, the NL float ambiguities derivated from the ionospheric-free float ambiguities in PPP and the WL integer values, are directly used in the FCB estimator. The low accuracy of the NL float ambiguities degrades the performance of FCB estimation. The ambiguity-fixed solutions from a GNSS network, where their double-difference ambiguities are got, are used for the NL FCB estimation (Geng et al. 2012). Additionally, the Kalman filter is also adopted for the FCB estimation epoch by epoch, which significantly speeds up the computation and is suitable for real-time applications (Xiao et al. 2018).

Distinguished from the FCB model proposed by Ge et al. (2008) and Laurichesse et al. (2009) presented the integer phase clock model, which consists of the ionospheric-free combination and the MW combination (Laurichesse et al. 2009). The NL FCBs are not estimated but assimilated into the estimates of satellite clock offsets. Hence, the NL ambiguities in the network solutions are directly fixed to the nearest integers, and the FCBcontained clock products based on the ambiguity-fixed solutions are determined (Laurichesse 2011). Similarly, Collins et al. (2008) estimated the integer recovery clocks for pseudorange and phase measurements, which is named as the decoupled clock model (Collins et al 2008). In this model, the effects from time-varying parts of code biases on the fractional parts of ambiguities are rigorously considered. The integer recovery clocks proposed by Collins et al. (2008) and Laurichesse et al. (2009) are used to recover the integer characteristics for the NL float ambiguities in PPP for ambiguity resolution. These two methods assimilated the NL FCBs into clocks estimates, which increased the computation of clock products. In the FCB method, the original International GNSS Service (IGS) precise clock products are used to separate the FCBs from ambiguities in an independent processing. Hence, the FCB method is widely adopted in many studies.

Most of the FCB estimation results are derived with the ionospheric-free PPP model. Recently, the uncombined PPP model with raw pseudorange and phase observations has attracted a great attention. The uncombined PPP model can directly estimate the raw ambiguities on each frequency and is flexible for processing multi-frequency observations. Its performance was demonstrated in the single- or dual-frequency PPP and PPP-RTK (Real-Time Kinematic) (Wübbena et al. 2005; Li et al. 2011; Zhang et al. 2011; Chen et al. 2015; Teunissen and Khodabandeh 2015; Lou et al. 2016). Li et al. (2013) presented the FCB estimates with the uncombined PPP model using the GPS L1 and L2 raw observations, which improved the performance of generating FCBs, and consequently improved the positioning accuracy of the PPP AR (Li et al. 2013). Gu et al. (2015a) determined the FCBs in the WL and NL combinations using the uncombined PPP model and demonstrated the ionosphere characteristics in the PPP AR (Gu et al. 2015a). This approach is also used for the triple-frequency FCBs estimation of BeiDou-2 Navigation Satellite System (BDS-2) (Gu et al. 2015b; Li et al. 2018). Xiao et al. (2019) further presented a triple-frequency FCB model for the PPP ambiguity resolution with raw observations, which is verified with Galileo Navigation Satellite System (Galileo) and BDS-2. The initial positioning performance of the PPP AR with the uncombined model is also demonstrated (Wang et al. 2020).

For implementing the PPP AR, the Centre National d'Etudes Spatiales (CNES) in French and the School of Geodesy and Geomatics at Wuhan University (SGGWHU) have been routinely generating the integer recovery clocks and FCBs for public PPP applications (Loyer et al. 2012; Li et al. 2016). Recently, SGG presented the PPP AR with GPS, BeiDou Navigation Satellite System (BDS), Galileo, and Quasi-Zenith Satellite System (QZSS) with multi-GNSS FCBs using the precise satellite orbits and clocks from different IGS centers ( $\mathrm{Hu}$ et al. 2020). Geng et al. (2019b) proposed a modified phase clock/bias model to improve the PPP AR and provided the phase clocks and daily phase biases based on the Center for 
Orbit Determination in Europe (CODE) precise satellite orbit products to users who process the PPP with software "PRIDE PPP-AR" (Geng et al. 2019a, b). Hence, the generation of FCBs for the PPP AR must be an essential work for supporting PPP services.

The international GNSS Monitoring and Assessment

\section{Methodology}

\section{Ambiguity-float PPP}

In the uncombined PPP model, the ionospheric delays can be either estimated or corrected. The linearized observation equations for pseudorange and phase observations from satellite $s$ to receiver $r$ are described as:

$$
\left\{\begin{array}{l}
\Delta P_{r, f}^{q, s}=\boldsymbol{u}_{r}^{q, s} \cdot \Delta \boldsymbol{x}+c \cdot\left(\mathrm{d} t_{r}^{q}-\mathrm{d} t^{q, s}\right)+M_{r}^{q, s} \cdot Z_{r}+\gamma_{f}^{q} \cdot I_{r, 1}^{q, s}+d_{r, f}^{q}-d_{f}^{q, s}+\varepsilon_{P, f}^{q} \\
\Delta L_{r, f}^{q, s}=\boldsymbol{u}_{r}^{q, s} \cdot \Delta \boldsymbol{x}+c \cdot\left(\mathrm{d} t_{r}^{q}-\mathrm{d} t^{q, s}\right)+M_{r}^{q, s} \cdot Z_{r}-\gamma_{f}^{q} \cdot I_{r, 1}^{q, s}+\lambda_{f}^{q} \cdot N_{r, f}^{q, s}+b_{r, f}^{q}-b_{f}^{q, s}+\varepsilon_{L, f}^{q}
\end{array}\right.
$$

System (iGMAS), which is a scientific project initiated and led by China under the United Nations framework, provides the daily satellite precise products for PPP users to get ambiguity-float solutions (Jiao et al. 2012). To meet the requirement of stable and reliable positioning, the ambiguity-fixed solutions should be implemented with the corresponding FCB products. In our work, the uncombined PPP model is adopted to generate the FCBs with the final satellite orbit and clock corrections with GPS, Galileo, and BDS-2 observations at the BeiDou analysis and service center of Chang'an University, which is a member of the iGMAS. The uncombined PPP model is flexible for PPP processing with multi-frequency and multi-GNSS observations. The raw ambiguities are directly estimated in the PPP model and then combined to estimate the phase bias in the FCB estimator. The initial results of the FCB generation and the PPP AR with GPS/BDS-2/Galileo data are analyzed to present the ambiguity-fixed positioning performance based on the iGMAS.

This contribution presented the great advantages of the multi-GNSS PPP in terms of convergence speed and positioning accuracy. The PPP AR based on iGMAS products showed the potential for global users in single station positioning services. In this paper, the method for FCB estimation and the PPP AR are introduced in detail. The integer coefficients $(4,-3)$ and $(1,-1)$ are adopted to decorrelate the involving parameters, reducing the effects of ionospheric delays. Then, the processing strategies and experiment data are introduced. To evaluate the PPP AR performances, the precision of the estimated where $\Delta P_{r, f}^{q, s}$ and $\Delta L_{r, f}^{q, s}$ are the respective pseudorange and phase measurements on the frequency $f(f=1,2)$, from which the computed values are removed; $\boldsymbol{u}_{r}^{q, s}$ is the receiver-to-satellite unit vector; $\Delta \boldsymbol{x}$ is the vector of the receiver position corrections to its preliminary position; $\mathrm{d} t_{r}^{q}$ and $\mathrm{d} t^{q, s}$ are the receiver and satellite clock errors, respectively; $c$ is the light speed in vacuum; $M_{r}^{q, s}$ is the elevation-dependent mapping function for the tropospheric wet delay from the corresponding zenith one $Z_{r} ; I_{r, 1}^{q, s}$ is the ionospheric delay along the line-of-sight from a receiver to a satellite at the first frequency and $\gamma_{f}^{q}=\left(\lambda_{f}^{q} / \lambda_{1}^{q}\right)^{2} ; \lambda_{f}^{q}$ is the wavelength for the frequency $f$ of a GNSS $q ; N_{r, f}^{q, s}$ is the phase ambiguity; $d_{r, f}^{q}$ and $b_{r, f}^{q}$ are the receiver hardware delays of code and phase observations, respectively; $d_{f}^{q, s}$ and $b_{f}^{q, s}$ are the satellite hardware delays of code and phase observations, respectively; $\varepsilon_{P, f}$ and $\varepsilon_{L, f}$ are the code and phase measurement noises, respectively, which include the multipath effects (Shi and Gao 2014).

Considering that the ionospheric-free combined observations which contain the satellite code biases are adopted for satellite clock error (parameter) estimates, the satellite clock parameters can be denoted as:

$$
\mathrm{d} \tilde{t}^{q, s}=\mathrm{d} t^{q, s}+\gamma_{2}^{q} /\left(\gamma_{2}^{q}-1\right) \cdot d_{1}^{q, s}+d_{2}^{q, s} /\left(1-\gamma_{2}^{q}\right)
$$

Hence, the receiver clock parameter is denoted as:

$$
\mathrm{d} \tilde{t}_{r}^{q}=\mathrm{d} t_{r}^{q}+\gamma_{2}^{q} /\left(\gamma_{2}^{q}-1\right) \cdot d_{r, 1}^{q}+d_{r, 2}^{q} /\left(1-\gamma_{2}^{q}\right)
$$

The ionospheric delays and ambiguities are reparameterized as:

$$
\left\{\begin{array}{c}
\tilde{I}_{r, 1}^{q, s}=I_{r, 1}^{q, s}+\left(\mathrm{DCB}_{r}^{q}-\mathrm{DCB}^{q, s}\right) /\left(\gamma_{2}^{q}-1\right) \\
\tilde{N}_{r, f}^{q, s}=N_{r, f}^{q, s}+b_{r, f}^{q}-b_{f}^{q, s}-\left(\gamma_{2}^{q}+1\right) /\left(\gamma_{2}^{q}-1\right) \cdot\left(d_{r, 1}^{q}-d_{1}^{q, s}\right)+2 /\left(\gamma_{2}^{q}-1\right) \cdot\left(d_{r, 2}^{q}-d_{2}^{q, s}\right)
\end{array}\right.
$$

FCBs for the three GNSS systems is demonstrated. The daily and hourly positioning accuracy is analyzed in static and kinematic modes. Finally, conclusions and outworks are discussed. where $\mathrm{DCB}_{r}=d_{r, 1}-d_{r, 2}$ and $\mathrm{DCB}^{s}=d_{1}^{s}-d_{2}^{s}$ are the Differential Code Biases (DCBs) for respective receiver and satellite. After applying the satellite clock corrections, the observations for the uncombined PPP model with GPS, BDS-2, and Galileo data can be written as: 


$$
\begin{aligned}
& \left\{\begin{array}{l}
\Delta P_{r, f}^{\mathrm{G}, s}=\boldsymbol{u}_{r}^{\mathrm{G}, s} \cdot \Delta \boldsymbol{x}+c \cdot \mathrm{d} \tilde{t}_{r}^{\mathrm{G}} \quad+M_{r}^{\mathrm{G}, s} \cdot Z_{r}+\gamma_{f}^{\mathrm{G}} \cdot \tilde{I}_{r, 1}^{\mathrm{G}, s}+\varepsilon_{P_{,}, f}^{\mathrm{G}} \\
\Delta P_{r, f}^{\mathrm{C}, s}=\boldsymbol{u}_{r}^{\mathrm{C}, s} \cdot \Delta \boldsymbol{x}+c \cdot \mathrm{d} \tilde{t}_{r}^{\mathrm{C}}+c \cdot \mathrm{ISB}_{r}^{\mathrm{C}}+M_{r}^{\mathrm{C}, s} \cdot Z_{r}+\gamma_{f}^{\mathrm{C}} \cdot \tilde{I}_{r, 1}^{\mathrm{C}, s}+\varepsilon_{P, f}^{\mathrm{C}} \\
\Delta P_{r, f}^{\mathrm{E}, s}=\boldsymbol{u}_{r}^{\mathrm{E}, s} \cdot \Delta \boldsymbol{x}+c \cdot \mathrm{d} \tilde{t}_{r}^{\mathrm{G}}+c \cdot \mathrm{ISB}_{r}^{\mathrm{E}}+M_{r}^{\mathrm{E}, s} \cdot Z_{r}+\gamma_{f}^{\mathrm{E}} \cdot \tilde{I}_{r, 1}^{\mathrm{E}, s}+\varepsilon_{P, f}^{\mathrm{E}}
\end{array}\right. \\
& \left\{\begin{array}{l}
\Delta L_{r, f}^{\mathrm{G}, s}=\boldsymbol{u}_{r}^{\mathrm{G}, s} \cdot \Delta \boldsymbol{x}+c \cdot \mathrm{d} \tilde{t}_{r}^{\mathrm{G}} \quad+M_{r}^{\mathrm{G}, s} \cdot Z_{r}-\gamma_{f}^{\mathrm{G}} \cdot \tilde{I}_{r, 1}^{\mathrm{G}, s}+\lambda_{r}^{\mathrm{G}} \cdot \tilde{N}_{r, f}^{\mathrm{G}, s}+\varepsilon_{L, f}^{\mathrm{G}} \\
\Delta L_{r, f}^{\mathrm{C}, s}=\boldsymbol{u}_{r}^{\mathrm{C}, s} \cdot \Delta \boldsymbol{x}+c \cdot \mathrm{d} \tilde{t}_{r}^{\mathrm{G}}+c \cdot \mathrm{ISB}_{r}^{\mathrm{C}}+M_{r}^{\mathrm{C}, s} \cdot Z_{r}-\gamma_{f}^{\mathrm{C}} \cdot \tilde{I}_{r, 1}^{\mathrm{C}, s}+\lambda_{r}^{\mathrm{C}} \cdot \tilde{N}_{r, f}^{\mathrm{C}, s}+\varepsilon_{L, f}^{\mathrm{C}} \\
\Delta L_{r, f}^{\mathrm{E}, s}=\boldsymbol{u}_{r}^{\mathrm{E}, s} \cdot \Delta \boldsymbol{x}+c \cdot \mathrm{d} \tilde{t}_{r}^{\mathrm{G}}+c \cdot \mathrm{ISB}_{r}^{\mathrm{E}}+M_{r}^{\mathrm{E}, s} \cdot Z_{r}-\gamma_{f}^{\mathrm{E}} \cdot \tilde{I}_{r, 1}^{\mathrm{E}, s}+\lambda_{r}^{\mathrm{E}} \cdot \tilde{N}_{r, f}^{\mathrm{E}, s}+\varepsilon_{L, f}^{\mathrm{E}}
\end{array}\right.
\end{aligned}
$$

where $\mathrm{ISB}_{r}^{\mathrm{C}}$ and $\mathrm{ISB}_{r}^{\mathrm{E}}$ are the inter-system bias for BDS-2 and Galileo with respect to GPS, respectively. Normally, the ISBs are regarded as constant which reflects the stability of receiver hardware code delays. However, the datum biases of the satellite clocks between different GNSS systems are also assimilated into the ISBs. The stability of ISBs is strongly correlated to specific satellite clock products. Correspondingly, the variations of ISBs are regarded as a white noise process. All estimated parameters in the Multi-GNSS PPP model are expressed as vector $X$ : and $(1,-1)$, which have the low noise and long wavelengths, are selected to improve the accuracy of the estimated ambiguities and reduce the impacts of ionospheric errors (Li et al. 2018; Xiao et al. 2019). Hence, the ambiguities are combined as:

$$
\left[\begin{array}{l}
\tilde{N}_{r,(4,-3)}^{q, s} \\
\tilde{N}_{r,(1,-1)}^{q, s}
\end{array}\right]=\left[\begin{array}{ll}
4 & -3 \\
1 & -1
\end{array}\right]\left[\begin{array}{l}
\tilde{N}_{r, 1}^{s} \\
\tilde{N}_{r, 2}^{s}
\end{array}\right]
$$

Here, the combined ambiguities, $\tilde{N}_{r,(4,-3)}^{q, s}$ and $\tilde{N}_{r,(1,-1)}^{q, s}$, are defined as new NL and WL ambiguities. The corre-

$$
\mathbf{X}=\left[\Delta \boldsymbol{x}, c \cdot \mathrm{d} \tilde{t}_{r}^{\mathrm{G}}, c \cdot \mathrm{ISB}_{r}^{\mathrm{C}}, c \cdot \mathrm{ISB}_{r}^{\mathrm{E}}, Z_{r}, \tilde{\mathbf{I}}_{r, 1}^{\mathrm{G}}, \tilde{\mathbf{I}}_{r, 1}^{\mathrm{C}}, \tilde{\mathbf{I}}_{r, 1}^{\mathrm{E}}, \tilde{\mathbf{N}}_{r, f}^{\mathrm{G}}, \tilde{\mathbf{N}}_{r, f}^{\mathrm{C}}, \tilde{\mathbf{N}}_{r, f}^{\mathrm{E}}\right]^{\mathrm{T}}
$$

where $\tilde{\mathbf{I}}_{r, 1}$ denotes the vector of the ionospheric delay parameters for all observed satellites and $\tilde{\mathbf{N}}_{r, f}$ denotes the vector of the ambiguity parameters.

Additionally, the stations in a reference network are used to extract the float ambiguities for FCBs estimation. The stations' coordinates are fixed to their references which are obtained in IGS SINEX files or from the preliminary static PPP processing for the stations not listed in SNX files.

\section{Fractional cycle bias estimation}

The ambiguity parameter in Eq. (4) can be rewritten as:

$$
\left\{\begin{array}{l}
\tilde{N}_{r, f}^{q, s}=N_{r, f}^{q, s}+B_{r, f}^{q}-B_{f}^{q, s} \\
B_{r, f}^{q}=b_{r, f}^{q}-\left(\gamma_{2}^{q}+1\right) /\left(\gamma_{2}^{q}-1\right) \cdot d_{r, 1}^{q}+2 /\left(\gamma_{2}^{q}-1\right) \cdot d_{r, 2}^{q} \\
B_{f}^{q, s}=b_{f}^{q, s}-\left(\gamma_{2}^{q}+1\right) /\left(\gamma_{2}^{q}-1\right) \cdot d_{1}^{q, s}+2 /\left(\gamma_{2}^{q}-1\right) \cdot d_{2}^{q, s}
\end{array}\right.
$$

where $N_{r, f}^{q, s}$ is an integer value, $B_{r, f}^{q}$ and $B_{f}^{q, s}$ are the respective receiver and satellite FCBs. Due to a strong correlation between ionospheric delays and ambiguities, which can be seen from Eq. (6), the accuracy of the estimated ambiguities on each frequency will be degraded by ionospheric errors. The combinations with integers $(4,-3)$ sponding FCBs are reformed as:

$$
\left[\begin{array}{l}
B_{(4,-3)}^{q} \\
B_{(1,-1)}^{q}
\end{array}\right]=\left[\begin{array}{ll}
4 & -3 \\
1 & -1
\end{array}\right]\left[\begin{array}{l}
B_{1}^{q} \\
B_{2}^{q}
\end{array}\right]
$$

Since the ambiguities have the same structure in Eq. (7), the fractional parts of ambiguities can be formulated as:

$$
\Delta n=\tilde{N}_{r}^{s}-N_{r}^{s}=B_{r}-B^{s}
$$

where $\Delta n$ denotes the FCB measurement which is the fractional part of the real-value ambiguity solution $\tilde{N}_{r}^{s}$. $N_{r}^{s}$ presents the integer part of the real-value ambiguity, which contains the original integer ambiguity and the integer part of the code and phase delays from satellite to receiver; $B_{r}$ and $B^{s}$ are the FCBs for receiver $r$ and satellite $s$, respectively. All FCB measurements in Eq. (11) from a reference network of $m$ stations and $n$ satellites tracked can be expressed as: 


$$
\left[\begin{array}{c}
\Delta n_{1}^{1} \\
\vdots \\
\Delta n_{r}^{s} \\
\vdots \\
\Delta n_{m}^{n}
\end{array}\right]=\left[\begin{array}{cc}
\boldsymbol{R}_{1} & \boldsymbol{S}^{1} \\
\vdots & \vdots \\
\boldsymbol{R}_{r} & \boldsymbol{S}^{s} \\
\vdots & \vdots \\
\boldsymbol{R}_{m} & \boldsymbol{S}^{n}
\end{array}\right]\left[\begin{array}{c}
B_{1} \\
\vdots \\
B_{r} \\
\vdots \\
B_{m} \\
B^{1} \\
\vdots \\
B^{s} \\
\vdots \\
B^{n}
\end{array}\right]
$$

In the coefficient vector $\boldsymbol{R}_{r}$, the $r$ th element is 1 and the others are zero. In the coefficient vector $\boldsymbol{S}^{S}$, the $s$ th element is -1 and the others are zero. One satellite for each GNSS system and new combined WL or NL ambiguities, respectively, is selected as a datum whose FCB is fixed to zero for resolving the rank deficiency in Eq. (12).

For the multi-GNSS data, the FCBs of new WL and NL combinations can be estimated together with GPS/ BDS-2/Galileo. To reduce the high computation load, the FCBs of the WL and NL combinations are estimated system by system. The individual FCBs are recovered by the inverse operation in Eq. (10). The multi-GNSS FCBs are integrated in one file by the predefined formats. The detailed flowchart is presented in Fig. 1.

Firstly, the static Uncombined PPP (UPPP) is processed for each reference station with its coordinates fixed to

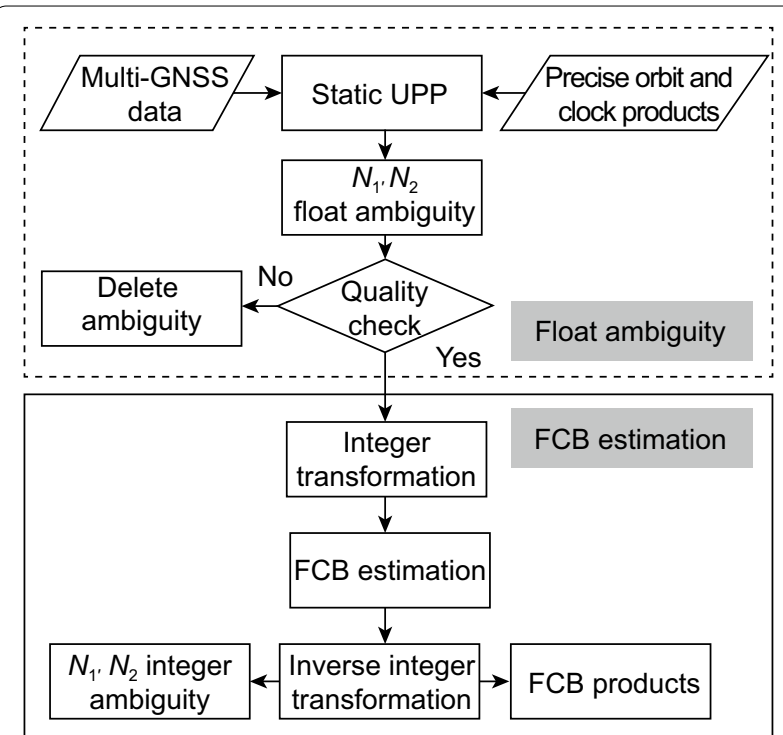

Fig. 1 Flowchart of FCB estimation using the individual ambiguities from the uncombined PPP model with the part of float ambiguity solutions in the red rectangle and the FCB estimation in the blue rectangle estimate the precise real-value ambiguities on each frequency of observed satellites. In this study, the FCBs are estimated with an interval of $15 \mathrm{~min}$. Hence, in the quality checking of the float ambiguity solutions, the ambiguities estimated with the observations of less than $10 \mathrm{~min}$ are deleted. After the quality checking, the ambiguities are inputted into the FCB estimator. Then, the individual ambiguities on each frequency are combined using Eq. (9). The FCBs measurements in Eq. (11) are adopted in the FCBs estimation for the WL and NL combinations. Finally, the inverse operation in Eq. (10) is conducted to recover the individual FCBs on each frequency. The raw FCB on each frequency is flexible in the State Space Representation (SSR) of Radio Technical Commission for Maritime service (RTCM) for users' PPP AR (Shi 2012).

\section{PPP AR at the user terminal}

After correcting the satellite FCBs for the estimation of float ambiguities, the SD PPP ambiguities are proposed in the ambiguity resolution for removing the receiver FCB. Additionally, the combined ambiguities, as shown in Eq. (9), are recommended for the FCB estimation. Hence, the WL and NL combinations of ambiguities are sequentially fixed. To eliminate the effects of measurement noise and multipath, the satellite elevation angle adopted in the ambiguity resolution should not be less than $15^{\circ}$. The ambiguity fixing success rate can be further improved by the partial ambiguity resolution method. Firstly, all available ambiguities are decorrelated. The reformed ambiguities are reordered in the ascending order according to their decorrelated variances. They can also be reordered according to the satellite elevation angles. Secondly, the Least-square AMBiguity Decorrelation Adjustment (LAMBDA) method is adopted to search for the optimal integer values. Thirdly, the bootstrapped success rate $P$ and the ratio test value $R$ are calculated. If $P<P_{0}$ or $R<R_{0}$, the last ambiguity with the lowest precision in the subset is removed and the second step is repeated. If the number of available ambiguities is less than four, the ambiguity-fixed solution fails. Otherwise, the integer ambiguities with a higher success rate and ratio value are confirmed as true values. Generally, the thresholds for $P_{0}$ and $R_{0}$ are set 0.999 and 2.0 (Li and Zhang 2015).

Once the integer values of ambiguities are confirmed, the tight constraint is imposed on the estimation of float ambiguities:

$$
0=\left(\tilde{N}_{r}^{q, s}-\tilde{N}_{r}^{q, n}\right)-\left(N_{r}^{q, s}-N_{r}^{q, n}\right)+\left(B_{r}^{q, s}-B_{r}^{q, n}\right)
$$

Using Eq. (13), the ambiguity-fixed solutions for positioning can be obtained after the PPP reprocessing. Note that the constraint of integer ambiguity can also increase the precision of other estimated parameters. 


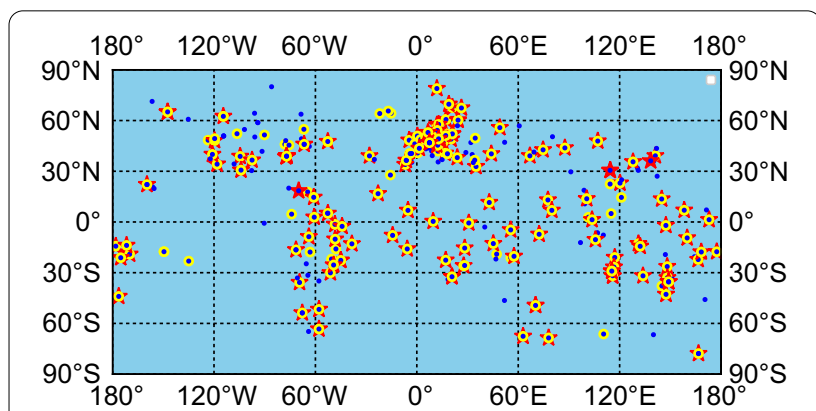

Fig. 2 Station distribution in the reference network for estimating FCBs. The blue points denote stations tracking GPS satellites. The red stars and yellow points are for BDS-2, Galileo, respectively

\section{Data and processing strategies}

To assess the performance of the FCB estimations with the satellite orbit and clock products at Chang'an University based on the iGMAS, 316 stations from iGMAS and Multi-GNSS EXperiment (MGEX) are selected, shown in Fig. 2.

GPS satellites are tracked at all stations, and Galileo satellites are tracked at 190 stations, among which 138 stations tracked BDS-2 satellites. The uncombined PPP is implemented with GPS, Galileo, and BDS-2 observations for extracting the float ambiguities as the measurements in the FCB estimation. After generating FCBs, the uncombined PPP AR is achieved for GPS/BDS-2/Galileo combined solutions. The elevation-dependent stochastic model $\sigma^{2}=\sigma_{0}^{2} / \sin ^{2}(\mathrm{el})$ is adopted for the GNSS observations, where $\sigma_{0}$ is $0.003 \mathrm{~m}$ and $0.3 \mathrm{~m}$ for carrier phase and code measurements, respectively, and el is the satellite elevation angle in radian. Among GPS, Galileo and BDS2 , the ratio of their observation noise is set as 1:1:3. The Phase Center Offset (PCO) and the Phase Center Variations (PCV) published by the European Space Agency (ESA) are used to correct the observations for BDS-2 satellites, while for the others the corrections are according to the IGS14.atx file. In PPP processing, the cutoff angle of satellite elevation is $7^{\circ}$ in the float solutions and $15^{\circ}$ in the AR, while it is $30^{\circ}$ in the FCB estimation for quality assurance. For the parameters estimated in PPP, the receiver clock and Inter-System Bias (ISB) are treated as white noise processes, the zenith tropospheric delay as a random walk process, and the ionospheric delays as white noise processes.

\section{Results and analysis}

The FCB products on Day of Year (DOY) 244, 2019 are generated to assess the performance of FCB estimation. Firstly, the stability of the FCB series over one day is analyzed with the averaged standard deviations for the combined FCBs and raw FCBs on each frequency. The accuracy of FCB estimation is evaluated by the posterior residuals of FCB measurements. After analyzing the performance of estimated FCBs, the uncombined PPP results are presented with the ambiguity-float and fixed solutions.

\section{FCB residuals distributions}

In the proposed method of FCB estimation, the FCBs for receiver and satellites are estimated separately using the WL and NL ambiguities. The FCB estimator directly provides the WL and NL combined FCBs. Hence, the accuracy assessment of the FCBs is carried out in the WL and NL combinations. The distribution of the posterior residuals is an intuitional indicator for the consistency of the FCBs results among different measurements. Generally, the residuals are close to zero indicating a high

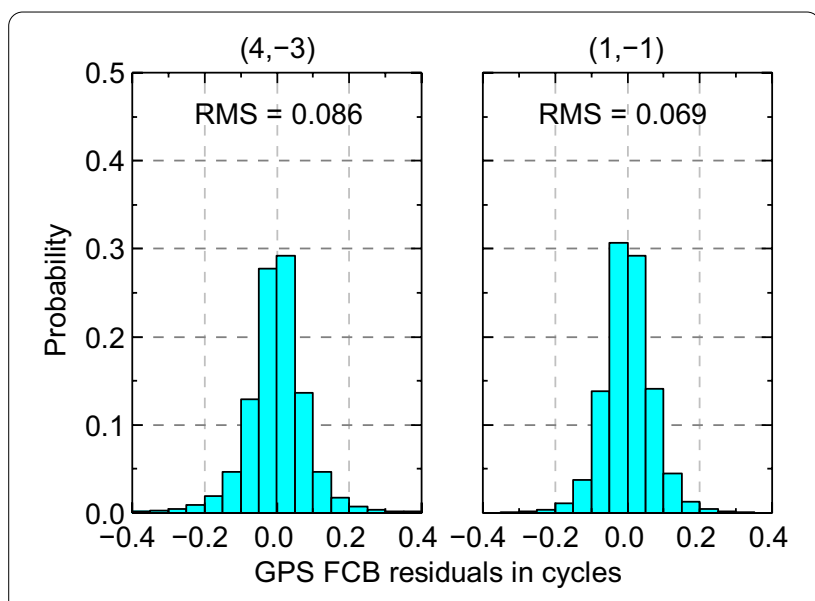

Fig. 3 Histogram of the GPS FCB residuals in the NL and WL linear combinations

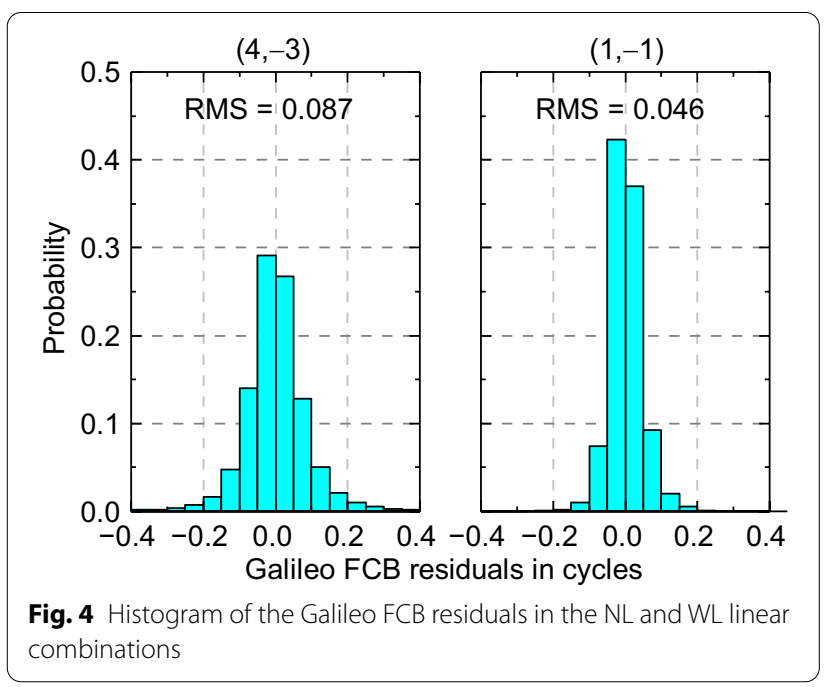




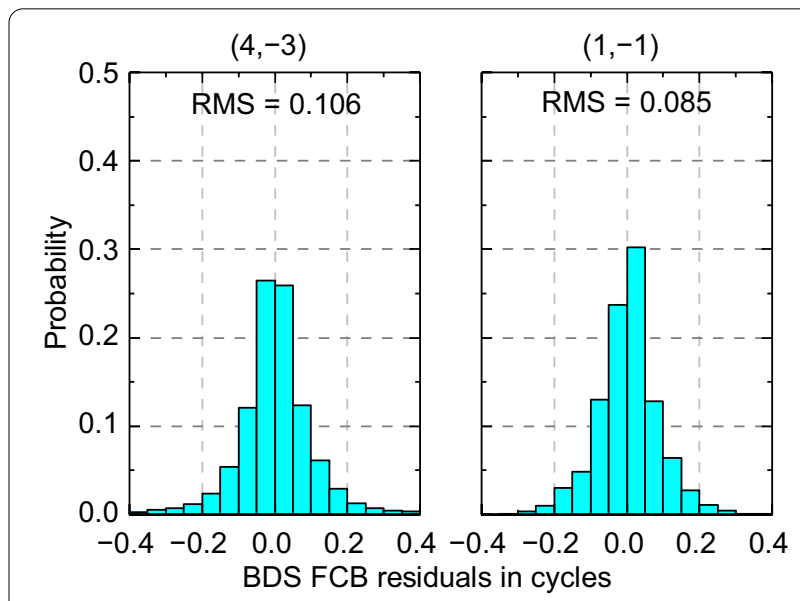

Fig. 5 Histogram of the BDS-2 FCB residuals in the NL and WL linear combinations

consistency of the estimated FCBs. Figures 3, 4 and 5 present the distribution of the posterior residuals in WL and NL linear combinations for GPS, Galileo, and BDS-2.

In the ionospheric-free model, the MW combination is commonly adopted to obtain the ambiguities for the WL FCB estimation, in which the accuracy of ambiguities is decreased by the averaging filter process. Compared with the FCB estimation in the ionospheric-free model, the WL combination reformed from the raw ambiguities on each frequency is free of pseudorange measurement noise and multipath. Generally, the residuals of the WL combination with longer wavelengths are smaller than the NL combination. For GPS satellites, the wavelength is about $86 \mathrm{~cm}$ for the WL combination and about $10 \mathrm{~cm}$ for the NL combination which is more sensitive to the errors. For GPS, Galileo, and BDS-2, this is verified by the RMS of the WL and NL residuals in Figs. 3, 4 and 5. The RMS of the WL residuals is $0.069,0.046$ and 0.085 cycles for GPS, Galileo, and BDS-2, while it is 0.086 , $0.087,0.106$ cycles for the NL residuals, respectively. The RMS of the residuals is around or less than 0.1 cycles, which indicates a high consistency among the estimated FCBs. Additionally, for the WL and NL residuals, the RMS for BDS-2 is larger than that for GPS and Galileo, which indicates that the accuracy of satellite orbit and clock products is crucial for the FCB estimation. For the NL combination, the RMS of GPS residuals is the smallest which is reasonable because of its precise ambiguity float solutions in PPP. For the distribution of the NL combination, $92.7 \%, 92.4 \%$, and $88.4 \%$ of the residuals are within $[-0.15,0.15]$ (in cycles) for GPS, Galileo and BDS-2, respectively, while that is $96.1 \%, 99.0 \%, 91.1 \%$ for the WL combination. The distributions also suggest that

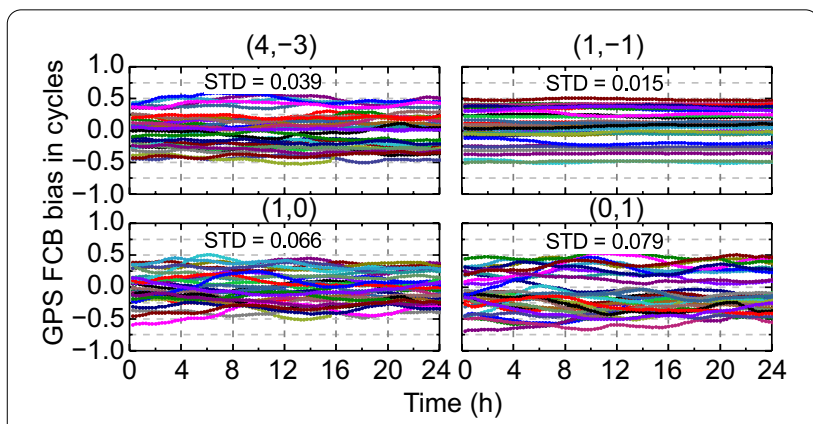

Fig. 6 GPS FCBs series for the $(4,-3),(1,-1)$ combinations, and individuals of each frequency. Each color denotes one satellite FCB series

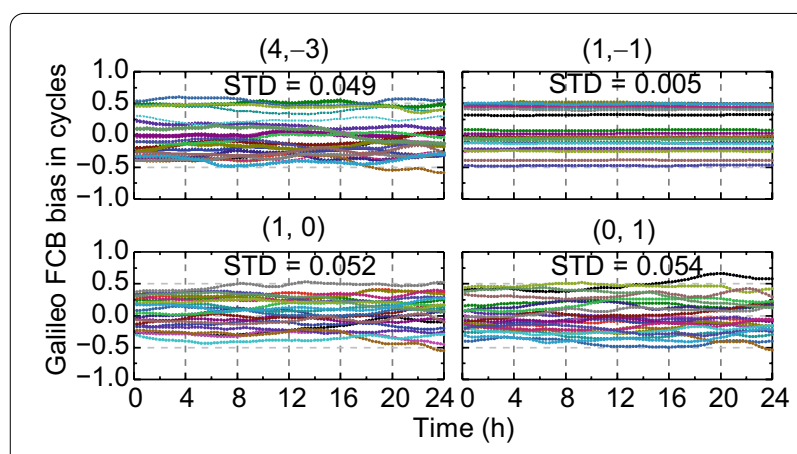

Fig. 7 Galileo FCBs series for the $(4,-3),(1,-1)$ combinations, and individuals of each frequency. Each color denotes one satellite FCB series

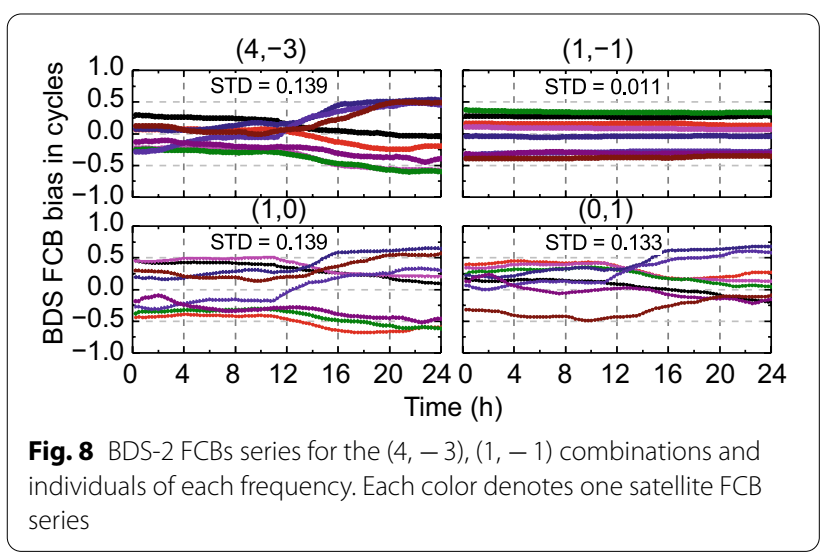

the high consistency of the FCB measurements ensures the good accuracy of FCB products.

\section{GNSS FCB series}

Figures 6, 7 and 8 show the one-day FCBs time series in the new WL and NL combinations and individuals on each frequency for respective GPS, Galileo, and BDS2. To further analyze the FCBs' stability, the STandard Deviation (STD) mean for all satellites is calculated. 
Compared with individual FCBs on each frequency, the stability is significantly better for the combined FCBs, especially for the WL combination. For the WL combination, the STD is $0.015,0.005,0.011$ cycles for respective GPS, Galileo, and BDS-2. The corresponding STD for the NL combination is $0.039,0.049,0.139$ cycles, which is larger than that for the WL combination. For individual FCB on each frequency, its fluctuation is remarkable with larger STDs. Hence, the more precise and stable FCB measurements from PPP processing are reasonable and more suitable for the FCB estimation.

For the BDS-2 shown in Fig. 8, the stability of the FCBs is much poorer than other systems except for the WL combinations. At present, the precision of BDS-2 satellite ephemeris is not as good as GPS or Galileo. Consequently, the accuracy of the float ambiguities in BDS-2 PPP is degraded and the RMS of residuals in BDS-2 FCB estimation is larger than other GNSS systems as shown in Fig. 5. Hence, the mean of STDs for the BDS-2 narrowlane FCBs is larger than 0.1 cycles.

\section{GPS/Galileo/BDS-2 PPP results}

The GPS/BDS-2/Galileo PPP performance is investigated by the ambiguity-float or -fixed solutions with our estimated FCB products. 24 stations in Fig. 9 from the iGMAS/MGEX network are selected to process the uncombined PPP and get the ambiguity resolutions with GPS, Galileo and BDS-2 dual-frequency observations. The daily static PPP solutions are presented to show the final positioning accuracy and the superiority of the PPP AR. Then, the hourly solutions are obtained to investigate the convergence time with different time lengths as well as the ambiguity success fixing rate. The kinematic PPP AR performance is also evaluated with daily observations.

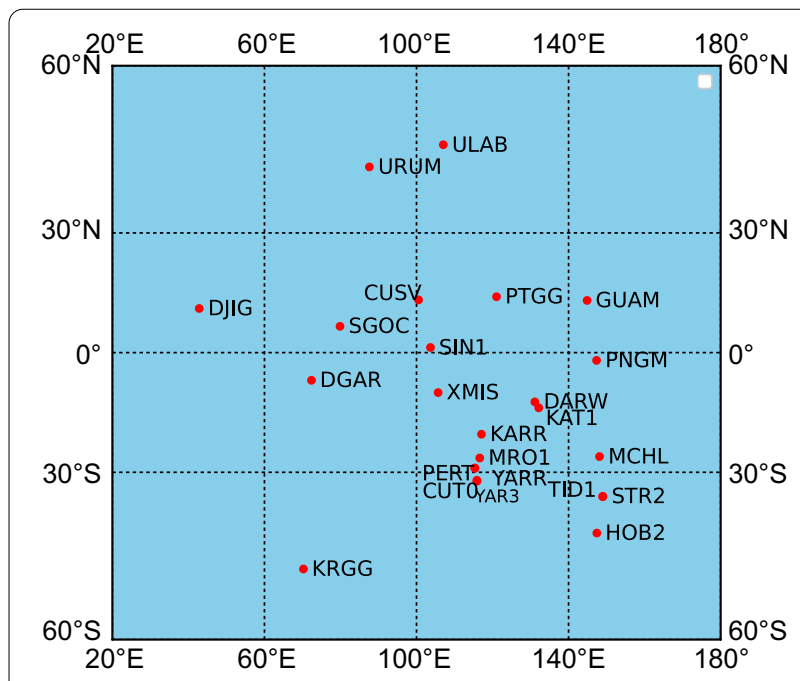

Fig. 9 Station distribution for PPP users with GPS, Galileo, and BDS-2

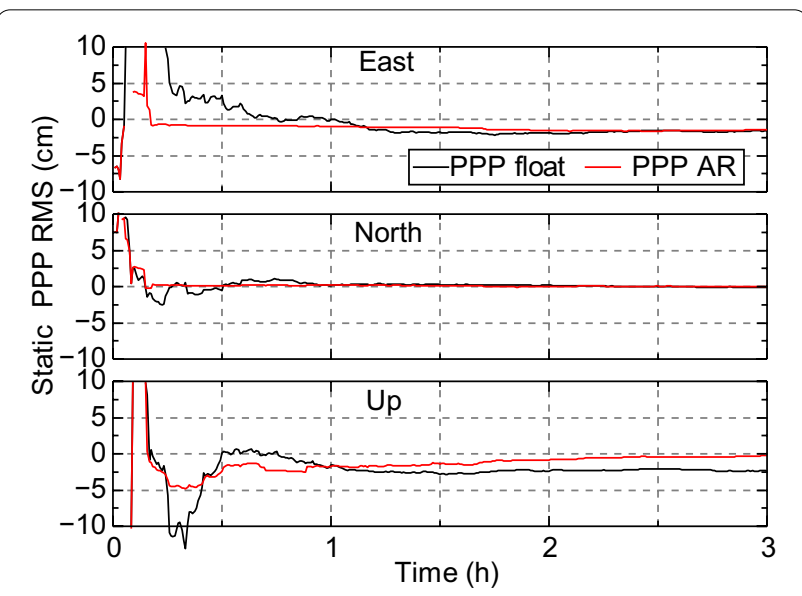

Fig. 10 Static PPP positioning errors for station YARR with 3-h data

Note that the reference coordinates for each station are the means of one-week static daily positioning solutions.

To study the stability of the PPP AR solutions, 3-h positioning errors from the one-day solution series at station YARR are presented in Fig. 10. Compared with the ambiguity-float solutions, the PPP AR achieves higher accuracy in a short period and gives more stable solutions. This indicates that the PPP AR can fast provide a reliable solution to users.

The RMS of the static daily positioning results for GPS/ BDS-2/Galileo PPP is shown in Fig. 11.

The RMS of the ambiguity-float solutions is 0.15, 0.06, and $0.20 \mathrm{~cm}$ for respective east, north, and up directions, while that is $0.14,0.06$, and $0.17 \mathrm{~cm}$ for the ambiguity-fixed solutions. The results indicate that the PPP

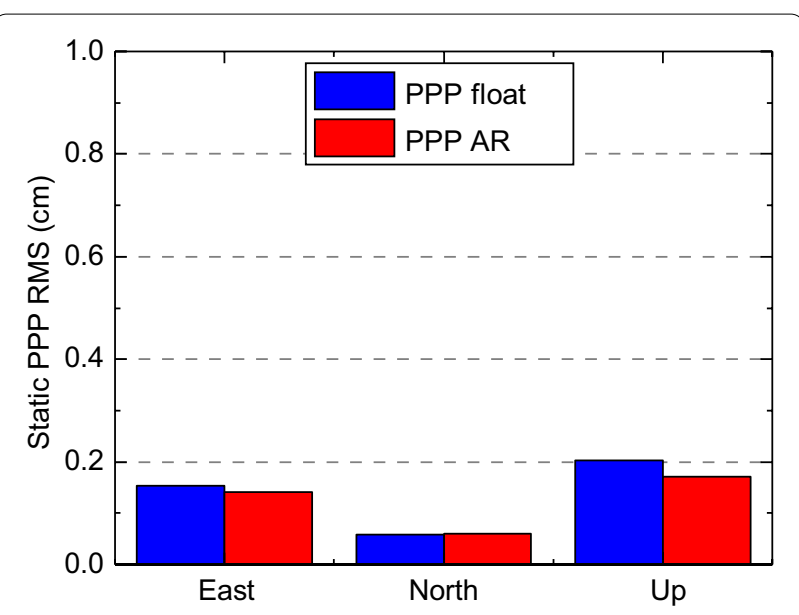

Fig. 11 Average static positioning RMS with 24-h GPS/BDS-2/Galileo data at 24 stations for the PPP float and AR solutions 


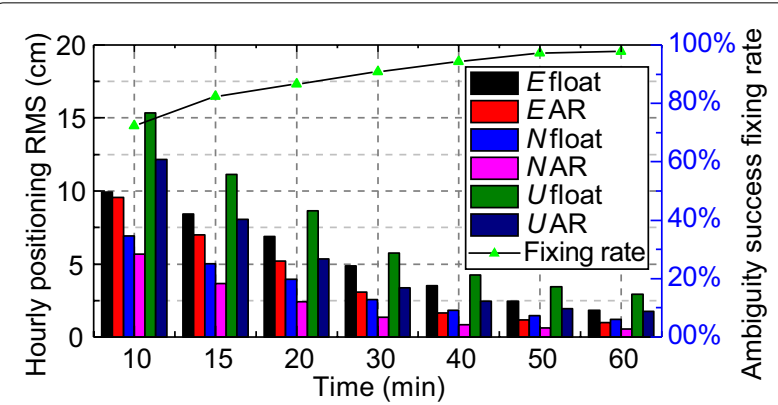

Fig. 12 Hourly static positioning errors and ambiguity success fixing rate

Table 1 Hourly static PPP positioning RMS and ambiguity success fixing rate

\begin{tabular}{llll}
\hline \multirow{2}{*}{ Items } & \multicolumn{3}{l}{ Results of different directions } \\
\cline { 2 - 4 } & East & North & Up \\
\hline RMS of float solutions (cm) & 1.83 & 1.21 & 2.93 \\
RMS of fixed solutions (cm) & 1.00 & 0.54 & 1.77 \\
Improvements rates (\%) & 45.5 & 55.1 & 39.7 \\
\hline
\end{tabular}

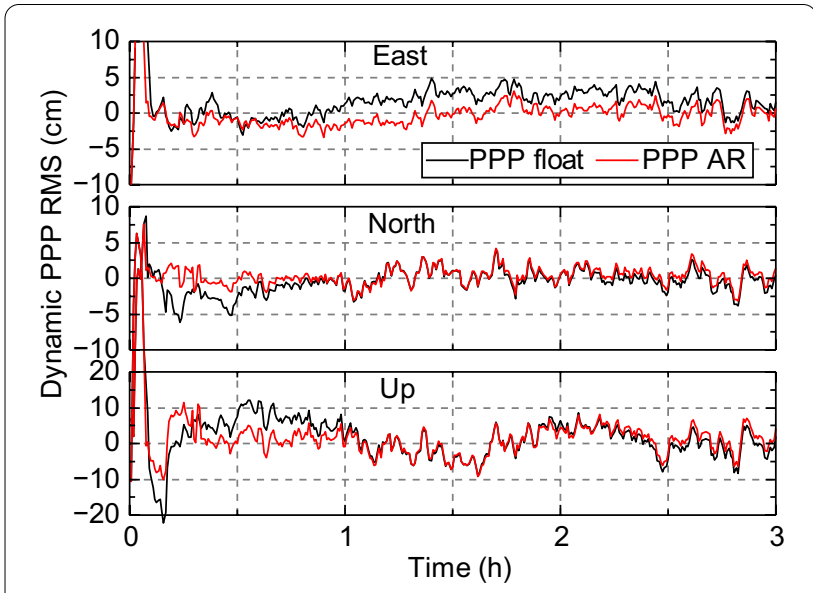

Fig. 13 Kinematic PPP positioning errors at YARR station

ambiguity-float and -fixed solutions have comparable accuracy with one-day observations.

Hourly solutions are obtained for the PPP AR in a short period of time, shown in Fig. 12.

The ambiguity success fixing rate is defined as the ratio of number of fixed solutions to that of all solutions. At different epochs, significant improvements in positioning RMS are achieved with the PPP AR. With the observations of $10 \mathrm{~min}$, the Three-Dimensional (3D) RMS is $12.72 \mathrm{~cm}$ for the ambiguity-float solutions, and $11.65 \mathrm{~cm}$ for the PPP AR. The ambiguity success fixing rate is only

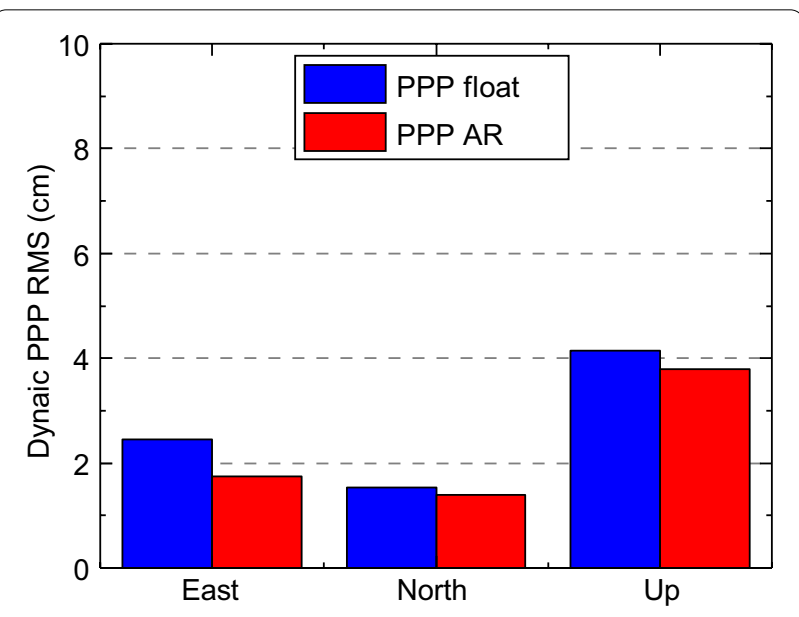

Fig. 14 Average position errors RMS of Kinematic PPP at 24 stations

Table 2 Average position errors RMS of Kinematic PPP at 24 stations

\begin{tabular}{llll}
\hline Items & \multicolumn{3}{l}{ Results of different directions } \\
\cline { 2 - 4 } & East & North & Up \\
\hline RMS of float solutions (cm) & 2.46 & 1.53 & 4.15 \\
RMS of fixed solutions (cm) & 1.75 & 1.39 & 3.79 \\
Improvements rates (\%) & 28.9 & 9.1 & 8.7 \\
\hline
\end{tabular}

72.3\%. For the observations of $15 \mathrm{~min}$, the 3D RMS is 10.37 and $8.41 \mathrm{~cm}$ for the respective ambiguity-float and -fixed solutions, and the ambiguity success fixing rate is $82.4 \%$. Finally, in hourly static solutions, the 3D RMS is $3.66 \mathrm{~cm}$ for ambiguity-float solutions and $2.10 \mathrm{~cm}$ for the PPP AR, improved by $42.6 \%$, and the ambiguity success fixing rate is $97.7 \%$. The positioning RMSs and improvements for respective east, north, and up directions are presented in Table 1.

The performance of the kinematic PPP with GPS/ BDS-2/Galileo data is also analyzed at the 24 stations. In Fig. 13, the initial 3-h kinematic positioning errors from the one-day solutions of station YARR show that the ambiguity-fixed solutions reduce significantly the fluctuation, realizing stable positioning services. In Fig. 14, the average position errors RMS of Kinematic PPP show that slight improvements are achieved for the PPP AR, compared with the ambiguity-float solutions. In Table 2, the RMS of kinematic mode is $2.46,1.53$, and $4.15 \mathrm{~cm}$ for the ambiguity-float solutions in respective east, north, and up directions, while that is $1.75,1.39$, and $3.79 \mathrm{~cm}$ for the PPP AR. The obvious improvement by $28.9 \%$ is in the east component. Hence, the kinematic PPP AR can 
improve 3D RMS by only $13.1 \%$ not as prominent as the static solutions.

\section{Conclusions}

To achieve the fast and precise single station positioning service, the PPP AR technique is the key to speed up the positioning bias convergence and improve the accuracy. Therefore, the FCB product is as essential as satellite orbit and clock products for PPP processing.

We first introduced the FCB estimation method with the uncombined PPP model and the PPP AR strategy. The precision of the FCBs products and the PPP AR solutions with GPS/BDS-2/Galileo are evaluated. The uncombined PPP method is used to estimate the raw ambiguities on each frequency which are for the FCBs estimation. Although we do not have to sequentially estimate the WL, NL FCBs as in the IF PPP model, the integer coefficients are used to reduce the correlation between the first two raw ambiguities in the uncombined PPP model. The more stable FCBs on the new WL and NL combinations which are formulated with integer vectors, $(1,-1)$ and $(4,-3)$, are used in the FCBs estimator. The raw FCBs on each frequency recovered from the new WL and NL combinations are provided to users to flexibly achieve the ambiguity-fixed solutions with a specific PPP model.

With the proposed method, we estimated the precise FCB products based on iGMAS satellite products. For the posterior residuals of the FCB estimation, the RMS of the WL combination is significantly smaller than the NL combination. The longer wavelength of the WL combination is helpful to resist errors and keep a high consistency among the FCB measurements. The accuracy of BDS FCBs is degraded by the lower accuracy of satellite precise products. This will be improved in the BeiDou-3 Navigation Satellite System (BDS-3).

After estimating the FCB products, the PPP AR with GPS, BDS-2, and Galileo raw observations are processed in static and kinematic modes. In daily static positioning, the ambiguity-fixed solutions have not significant improvements compared with the ambiguity-float solutions, while the stability and reliability of positioning are enhanced with the PPP AR which is also found in kinematic mode. With hourly static solutions, $42.6 \%$ improvement and $97.7 \%$ ambiguity success fixing rate are achieved for the ambiguity-fixed solutions compared with the float solutions. The significant improvement of faster convergence time of positioning is also found. Finally, the 3D RMS for the ambiguity-fixed solutions is $2.10 \mathrm{~cm}$.

Currently, only the BDS-2 data is adopted in our estimation which will be improved with the available BDS-3 satellites in the next work. The uncombined PPP model is flexible to process the triple-, quadruple-frequency or more frequency observables. Hence, the multi-frequency FCB products will be generated in future work.

Acknowledgements
Thanks to the iGMAS/MGEX for providing the data.

\section{Authors' contributions}

QZ, JW and GH conceived and designed the experiments. JW and GH evaluated the accuracy of products and wrote the paper. All authors read and approved the final manuscript.

\section{Funding}

The National Key Research and Development Program of China (2018YFC1505102), the Programs of the National Natural Science Foundation of China $(41774025,41731066)$, the Special Fund for Technological Innovation Guidance of Shaanxi Province (2018XNCGG05), the Special Fund for Basic Scientific Research of Central Colleges (CHD300102269305, CHD300102268305), the Grand Projects of the BDS-2 System (GFZX0301040308) supported this study.

\section{Availability of data and materials}

The data used in this study is obtained from iGMAS (http://www.igmas.org) and MGEX (ftp://ftp.cddis.eosdis.nasa.gov/pub/gnss/data/). The satellite precise orbit and clock products are issued by Chang'an Universtiy (http://gnssc enter.chd.edu.cn).

\section{Competing interests}

The authors declare no conflicts of interest.

Received: 24 August 2020 Accepted: 2 February 2021

Published online: 17 May 2021

\section{References}

Bisnath, S., \& Gao, Y. (2008). Current state of precise point positioning and future prospects and limitations. In M. G. Sideris (Ed.), Observing our changing Earth (pp. 615-623). Berlin, Heidelberg: Springer.

Chen, J. P., Zhang, Y. Z., Wang, J. G., Yang, S. N., Dong, D. N., Wang, J. X., et al. (2015). A simplified and unified model of multi-GNSS precise point positioning. Advances in Space Research, 55(1), 125-134. https://doi. org/10.1016/j.asr.2014.10.002.

Collins, P., Lahaye, F., Héroux, P., \& Bisnath, S. (2008). Precise point positioning with ambiguity resolution using the decoupled clock model. In Proceedings of the 21st international technical meeting of the satellite division of the Institute of Navigation (pp. 1315-1322). Savannah, GA, USA: ION.

Gabor, M.J., \& Nerem, R.S. (1999). GPS carrier phase ambiguity resolution using satellite-satellite single differences. In Proceedings of the 12th international technical meeting of the satellite division of the Institute of Navigation (pp. 1569-1578). Nashville, TN, USA: ION.

Ge, M., Gendt, G., Rothacher, M., Shi, C., \& Liu, J. (2008). Resolution of GPS carrier-phase ambiguities in Precise Point Positioning (PPP) with daily observations. Journal of Geodesy, 82(7), 389-399. https://doi.org/10.1007/ s00190-007-0187-4

Geng, J. H., Chen, X. Y., Pan, Y. X., Mao, S. Y., Li, C. H., Zhou, J. N., \& Zhang, K. L. (2019a). PRIDE PPP-AR: An open-source software for GPS PPP ambiguity resolution. GPS Solutions, 23(4), 91. https://doi.org/10.1007/s1029 1-019-0888-1.

Geng, J. H., Chen, X. Y., Pan, Y. X., \& Zhao, Q. L. (2019b). A modified phase clock/ bias model to improve PPP ambiguity resolution at Wuhan University. Journal of Geodesy, 93(10), 2053-2067. https://doi.org/10.1007/s0019 0-019-01301-6.

Geng, J. H., Shi, C., Ge, M. R., Dodson, A. H., Lou, Y. D., Zhao, Q. L., \& Liu, J. N. (2012). Improving the estimation of fractional-cycle biases for ambiguity resolution in precise point positioning. Journal of Geodesy, 86(8), 579-589. https://doi.org/10.1007/s00190-011-0537-0.

Gu, S. F., Lou, Y. D., Shi, C., \& Liu, J. N. (2015b). BeiDou phase bias estimation and its application in precise point positioning with triple-frequency observable. Journal of Geodesy, 89(10), 979-992. https://doi.org/10.1007/s0019 0-015-0827-z. 
Gu, S. F., Shi, C., Lou, Y. D., \& Liu, J. N. (2015a). Ionospheric effects in uncalibrated phase delay estimation and ambiguity-fixed PPP based on raw observable model. Journal of Geodesy, 89(5), 447-457. https://doi.org/10.1007/ s00190-015-0789-1.

Hu, J. H., Zhang, X. H., Li, P., Ma, F. J., \& Pan, L. (2020). Multi-GNSS fractional cycle bias products generation for GNSS ambiguity-fixed PPP at Wuhan University. GPS Solutions, 24(1), 15. https://doi.org/10.1007/s10291-019-0929-9.

Jiao, W. H., Ding, Q., Li, J. W., Lu, X. C., Feng, L. P., Ma, J. Q., \& Chen, G. (2012). Monitoring and assessment of GNSS Open Services (in Chinese). Science Sinica Physics, Mechanics \& Astronomy, 41(5), 521-527. https://doi. org/10.1360/132011-359.

Laurichesse, D. (2011). The CNES Real-time PPP with undifferenced integer ambiguity resolution demonstrator. In Proceedings of the 24th international technical meeting of the satellite division of the institute of navigation (pp. 654-662). Portland: ION.

Laurichesse, D., Mercier, F., Berthias, J.-P., Broca, P., \& Cerri, L. (2009). Integer ambiguity resolution on undifferenced GPS phase measurements and its application to PPP and satellite precise orbit determination. Navigation, 56(2), 135-149. https://doi.org/10.1002/j.2161-4296.2009.tb01750.x.

Li, P., \& Zhang, X. H. (2015). Precise point positioning with partial ambiguity fixing. Sensors, 15(6), 13627-13643. https://doi.org/10.3390/s150613627.

Li, P., Zhang, X. H., Ge, M. R., \& Schuh, H. (2018). Three-frequency BDS precise point positioning ambiguity resolution based on raw observables. Journal of Geodesy, 92(12), 1357-1369. https://doi.org/10.1007/s0019 0-018-1125-3.

Li, P., Zhang, X. H., Ren, X. D., Zuo, X., \& Pan, Y. M. (2016). Generating GPS satellite fractional cycle bias for ambiguity-fixed precise point positioning. GPS Solutions, 20(4), 771-782. https://doi.org/10.1007/s10291-015-0483-z.

Li, X. X., Ge, M. R., Zhang, H. P., \& Wickert, J. (2013). A method for improving uncalibrated phase delay estimation and ambiguity-fixing in real-time precise point positioning. Journal of Geodesy, 87(5), 405-416. https://doi org/10.1007/s00190-013-0611-x.

Li, X. X., \& Zhang, X. H. (2012). Improving the estimation of uncalibrated fractional phase offsets for PPP ambiguity resolution. Journal of Navigation, 65(3), 513-529. https://doi.org/10.1017/S0373463312000112.

Li, X. X., Zhang, X. H., \& Ge, M. R. (2011). Regional reference network augmented precise point positioning for instantaneous ambiguity resolution. Journal of Geodesy, 85(3), 151-158. https://doi.org/10.1007/s0019 0-010-0424-0.

Lou, Y. D., Zheng, F., Gu, S. F., Wang, C., Guo, H. L., \& Feng, Y. M. (2016). MultiGNSS precise point positioning with raw single-frequency and dualfrequency measurement models. GPS Solutions, 20(4), 849-862. https:// doi.org/10.1007/s10291-015-0495-8.

Loyer, S., Perosanz, F., Mercier, F., Capdeville, H., \& Marty, J.-C. (2012). Zero-difference GPS ambiguity resolution at CNES-CLS IGS Analysis Center. Journal of Geodesy, 86(11), 991-1003. https://doi.org/10.1007/s00190-012-0559-2.
Melbourne, W.G. (1985). The case for ranging in GPS-based geodetic systems. In Proceedings of the First international symposium on precise positioning with the Global Positioning System (pp. 373-386). Rockville: U.S. Dept. of Commerce.

Shi, J.B. (2012). Precise point positioning integer ambiguity resolution with decoupled clocks. Ph.D. thesis. Calgary: University of Calgary.

Shi, J. B., \& Gao, Y. (2014). A comparison of three PPP integer ambiguity resolution methods. GPS Solutions, 18(4), 519-528. https://doi.org/10.1007/ s10291-013-0348-2.

Teunissen, P. J. G., \& Khodabandeh, A. (2015). Review and principles of PPP-RTK methods. Journal of Geodesy, 89(3), 217-240. https://doi.org/10.1007/ s00190-014-0771-3.

Wang, J., Huang, G. W., Yang, Y. X., Zhang, Q., Gao, Y., \& Xiao, G. R. (2019). FCB estimation with three different PPP models: equivalence analysis and experiment tests. GPS Solutions, 23(4), 93. https://doi.org/10.1007/s1029 1-019-0887-2.

Wang, J., Huang, G. W., Zhang, Q., Gao, Y., Gao, Y. T., \& Luo, Y. R. (2020). GPS/ BDS-2/Galileo precise point positioning ambiguity resolution based on the uncombined model. Remote Sensing, 12(11), 1853. https://doi. org/10.3390/rs12111853.

Wübbena, G. (1985). Software developments for geodetic positioning with GPS using TI 4100 code and carrier measurements. In Proceedings 1st international symposium on precise positioning with the Global Positioning System (pp. 403-412). Rockville, Maryland: U. S. Dept. of Commerce.

Wübbena, G., Schmitz, M., \& Bagge, A. (2005). PPP-RTK: precise point positioning using state-space representation in RTK networks. In Proceedings of the 18th international technical meeting of the satellite division of the Institute of Navigation (pp. 13-16). Long Beach: ION.

Xiao, G. R., Li, P., Gao, Y., \& Heck, B. (2019). A unified model for multi-frequency PPP ambiguity resolution and test results with galileo and Beidou triple-frequency observations. Remote Sensing, 11(2), 116. https://doi. org/10.3390/rs11020116.

Xiao, G. R., Sui, L. F., Heck, B., Zeng, T., \& Tian, Y. (2018). Estimating satellite phase fractional cycle biases based on Kalman filter. GPS Solutions, 22(3), 82. https://doi.org/10.1007/s10291-018-0749-3.

Zhang, B. C., Teunissen, P. J. G., \& Odijk, D. (2011). A novel un-differenced PPP-RTK concept. Journal of Navigation, 64(S1), S180-S191. https://doi. org/10.1017/S0373463311000361.

\section{Publisher's Note}

Springer Nature remains neutral with regard to jurisdictional claims in published maps and institutional affiliations.

\section{Submit your manuscript to a SpringerOpen ${ }^{\circ}$ journal and benefit from:}

- Convenient online submission

- Rigorous peer review

- Open access: articles freely available online

- High visibility within the field

- Retaining the copyright to your article

Submit your next manuscript at $\boldsymbol{\Delta}$ springeropen.com 